\section{Behavior of Particle Size Distributions, Means and BET Values in Ideal and Non- Ideal Morphology Systems in a TEM}

\author{
Paul Beauregard \\ Chemist and Electron Microscopist \\ Beaurega@westol.com
}

Historically, one of the first jobs performed by the new instrument called a "transmission electron microscope" (TEM) was the determination of particle size and distribution. Particle size is still important in controlling the properties of sub-micron and nanoparticle products. The width and average particle size can determine the light scattering properties of a pigment like $\mathrm{TiO}_{2}$, for example. The cost of installing a TEM facility and TEM sample preparation issues makes TEM analysis expensive and labor intensive. So decades ago, nitrogen BET (Brunauer, Emmett, and Teller) surface area determinations were used on sub-micron powders and pigments to minimize these costs. The BET measurement of the

\section{Primary Particle and Reinforcement Morphologies (Added Surface Area and Volume Shown in White)}

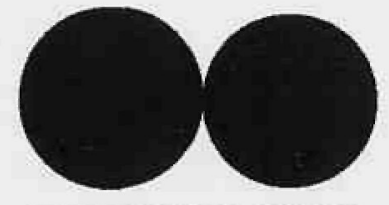

2 MONODISPERED SPHERES

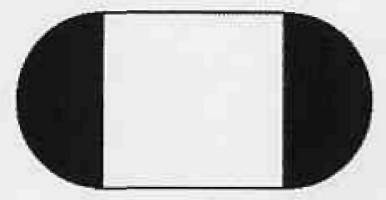

$100 \%$ REINFORCEMENT

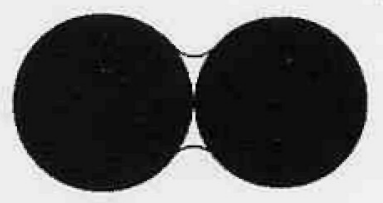

$50 \%$ REINFORCEMENT

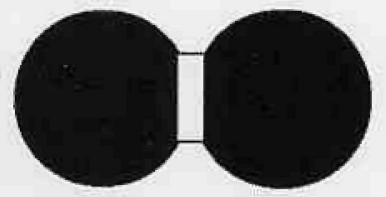

$50 \%$ REINFORCEMENT

\section{FIGURE 1}

TOP-LEFT: Two point touching monodispersed primary particles.

TOP-RIGHT: Two real world primary particles, reinforcement shown in white. BOTTOM-RIGHT: Cylinder \& Sphere Mathematical Model

BOTTOM-LEFT: $100 \%$ Reinforcement between primary particles.

exposed external surface area of a material is made by detecting and measuring the amount of nitrogen given off after absorption, assuming one atomic layer of nitrogen is adsorbed. The problem is that BET tells you nothing of the size distribution or of morphology changes. Sub-micron manufacturing facilities can inexpensively perform BET measurements and avoid the overhead of a TEM and lab. However, when something goes wrong, and BET does not show any differences, one needs to use a TEM. For this reason, a skilled microscopist is needed to understand the relationships between BET and TEM measurements to determine what the problem is. This subject is not new but this article is a primer on that BET/TEM relationship. We'll look at the effects of primary particle morphologies seen in a TEM, compared to BET surface area, and other techniques like laser diffraction.

\section{First Principles and Monodispersed Systems}

If one thinks about the interrelationship between the absolute or true surface area of a solid sphere and its diameter, one sees that the relationship is an inverse mathematical one. If the diameter size decreases, then the surface area will increase.

We will initially assume that the nanometer-scale primary particles in this article are molecularly smooth-surfaced spheres, are rigid, are of equal diameter (monodispersed) and that they only point touch when they are 'coagulated'. This is the simple relationship we would like to explore. See Figure 1.

Let's look at the ratio of absolute surface area of a sphere to its volume and it's density.

From the ratio of these two we get:

$$
\frac{S A}{V o l}=S A P V=\frac{\pi d^{2}}{\frac{\pi d^{3}}{6}}=\frac{6 d^{2}}{d^{3}}=\frac{6}{d}
$$

To include a term for density, $\rho$, we multiply by $1 / \rho$ :

$$
\frac{S A}{\text { Volume } \times \text { Density }}=\frac{6}{\rho d}
$$

For comparisons, we want the BET nitrogen specific surface area (SAPG) in meters ${ }^{2}$ per gram and the TEM primary particle diameters in terms of nanometers ( $\mathrm{nm}$ ).

$$
S A P G=\frac{6 \pi d^{2}\left(\mathrm{~nm}^{2}\right) \times\left(\mathrm{m}^{3}\right) \times 10^{9} \mathrm{~nm}}{\pi d^{3}\left(\mathrm{~nm}^{3}\right) \times \rho\left(\mathrm{g} / \mathrm{cm}^{3}\right) \times 10^{6}\left(\mathrm{~cm}^{3}\right) \times(\mathrm{m})}
$$

Simplifying and rearranging, we get:

$$
d \times S A P G=d \times \mathrm{BET}=\frac{6000}{\rho}
$$

Where density $P$ is in grams $/ \mathrm{cm}^{3}$, the diameter $d$ is in nanometers and $S A P G$ is in meters ${ }^{2}$ per gram.

In equation (3), d is really the mean surface volume diameter (MSVD). So the diameter times SAPG (or BET) is a constant at any density of primary particles. Equation (3) shows that the surface area per gram (BET) decreases as the diameter increases.

Mean surface volume diameter is the average diameter value of a hypothetical monodispersed system that has the same surface to volume ratio as the actual polydispersed system we measured by BET but, it does not exist and we didn't count a monodispersed system. The BET MSVD calculated value does not have any particle size distribution (PSD) data with it, and so, this MSVD value represents a single frequency diameter value. The calculated value of MSVD from BET and any other MSVD value without any PSD data associated with them, represents a hypothetical monodispersed system.

On the other hand, one can calculate a TEM MSVD value from the TEM PSD data we measured on the same polydispersed system. This value represents a polydispersed system, but if we remove the PSD by frequency data, then it also represents a hypothetical monodispersed system. This TEM MSVD value can equal the BET MSVD (monodispersed) or not (non-ideal polydispersed). This distinction is important in the calculations of particles per unit volume mentioned below.

In a monodispersed system involving rigid spheres, regular shapes, no microporosity, no intergrowth and equal sized spheres, the TEM MSVD, the TEM number mean, the CTAB ${ }^{2}$ MSVD and the BET MSVD are all equal. Why? Every perfectly solid and rigid sphere is equal in size and shape. So all the different mean numbers are equal. 


\section{Piecing Together Analytical Solutions}
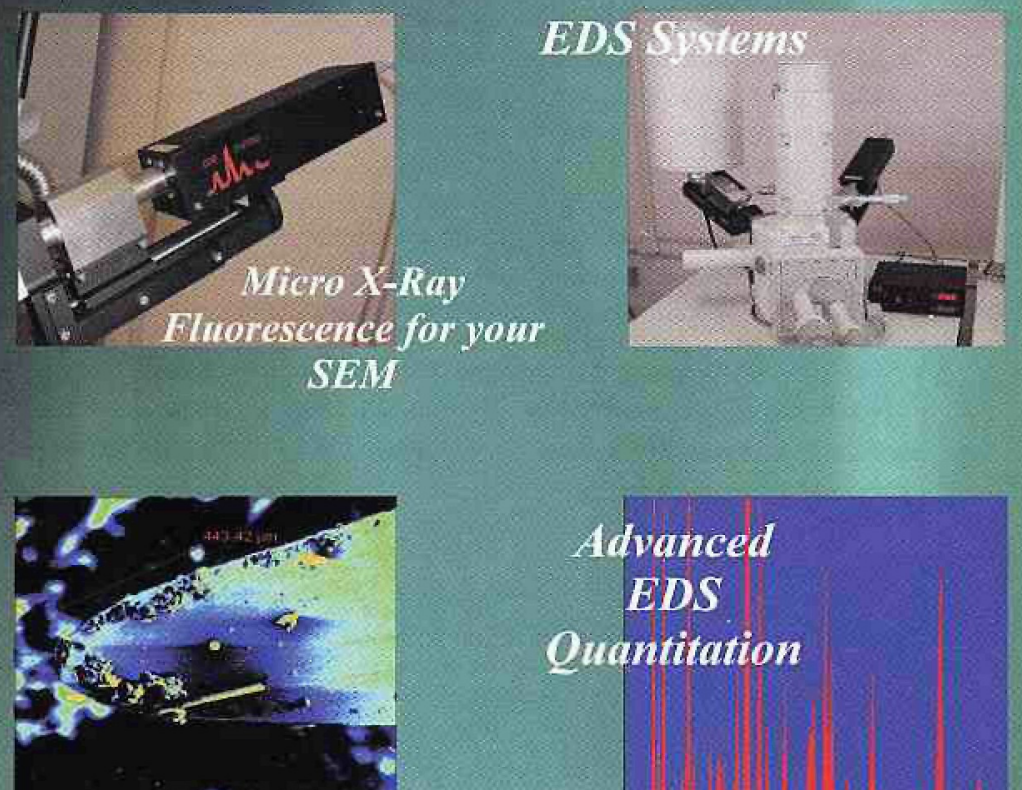

Digital Imaging and Advanced Feature Measumments

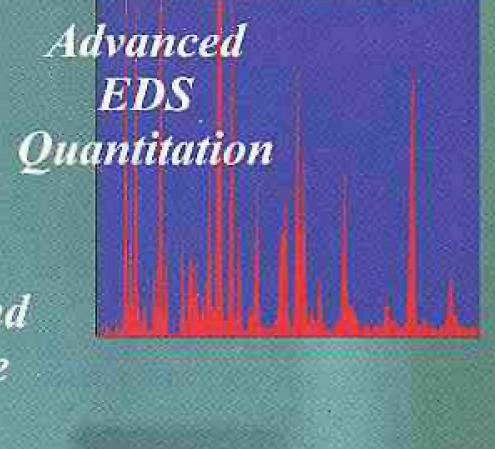

Whether it is Digital Image Analysis X-ray Mapping or Quantitative EDS you are looking for, IXRF has your solution. Designed to meet the analytical needs of you, the user, simple yet powerful tools provide a price to performance ratio that is unparalleled.

- Flexible, full customization throughout the software

Comprehensive, adaptive spectrum processing and the highest level of quantitative analysis

- Powerful, antomated, and complete statistical digital image analysis that includes Feature Analysis on phases or particles

Q Highly interactive, showease, fullspectrum $x$-ray mapping that includes fractionated phase or chemical composition statistical analysis

Remember: As evidence of our commitment to our customers, IXRF will provide Free software upgrades for as long as you own your system. Our software is improved and upgraded largely based on customer suggestions.

But this should be old news. Over the past decade IXRF has fundamentally changed the industry. As another example of our dedication to innovtion, we are offering, with an industry first, the addition of small-spot XRP analysis to the world of Scanning Dlectron Microscopy. Experience elemental sensitivities and detection limits that are 10100 times greater than electron beam excitation. Imagine looking at a sample and seeing 5 elemental peaks. NOW, imagine "discovering" an additional 25 peaks!!

We pride ourselves in eustomer service. Call us at (281) 286-6485 to talk to any of our staff, or ask one of our hundreds of customers- after all- they're our best sales people!
IXRF Systems, lnc.

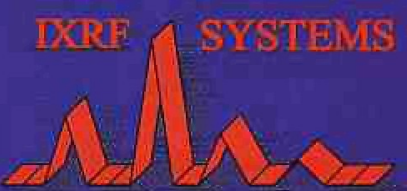




\section{An Ideal Polydispersed Primary Particle System}

Now let's introduce a polydispersed system meaning that we will have a wider primary particle size distribution. In this polydispersed system, we will only change the width of the particle size distribution. All the other assumptions are still in effect except the sizes are not equal. The only mean value to change in this special system will be the TEM number mean and it will be less than any MSVD. Polydispersed systems must involve more complex calculations than those based on simple, single-sized geometric solids or a macro-determination by BET.

The TEM number mean is simply the sum of the addition of all the individual diameters $\left(d_{i}\right)$ of the primary particles measured divided by the total number of counts $(N)$.

$$
d=\sum_{i} \frac{d_{i}}{N}
$$

Now when measuring the diameters interactively using an image analysis program on-line with a TEM, some diameters will be duplicated. So, we must change the above formula to reflect the frequency of any one diameter. So a more correct formula ${ }^{3}$ would be,

$$
d=\frac{\sum_{i} n_{i} d_{i}}{\sum_{i} n_{i}}
$$

The TEM mean surface volume diameter is:

$$
\text { MSVID TEM }_{1}=\frac{\sum_{i} n_{i} d_{i}^{3}}{\sum_{i} n_{i} d_{i}^{2}}
$$

The area per unit volume (APUV) is the sum of the SA to VOL ratios:

$$
\frac{S A}{V O L}=\frac{6 \sum_{1} n_{i} d_{i}^{2}}{\sum_{i} n_{i} d_{i}^{3}}
$$

Notice that (4) has the same form as equation (1), 6/d, in the monodispersed world.

The number of particles per unit volume is:

$$
\text { PPUV }=\frac{N}{\sum_{i} n_{i} \times\left(\begin{array}{l}
\pi \\
6
\end{array}\right) \times d_{i}}
$$

The number of particles per unit volume, PPUV, should be a measure of the number of particles of the actual polydispersed system's PSD in a cubic micrometer. This number tells us the number of primary particles being produced by the reactor at its output per cubic micron. This should be an important measure of what is going on when one makes nanoparticles in a plasma reactor. The MSVD calculated from BET will not yield the correct number for PPUV.

In this special polydispersed system, the BET, TEM and CTAB MSVDs will all be equal. The deviation in the TEM number mean from the MSVD value is a measure of the width of the distribution. So too is the standard deviation of the mean of the various diameters measured in the TEM. This deviation can also be a measure of the degree of outliers or large reactor growth units in a system like a plasma reactor making nanoparticles.
Here are some typical numbers for a nanoparticle sample obtained from real TEM diameter measurements and a real polydispersed BET determination:

$\begin{array}{lr}\text { TEM number mean: } & 40 \mathrm{~nm} \text { (360KX) } \\ \text { TEM calc'd MSVD: } & 60 \mathrm{~nm} \\ \text { TEM calc'd BET: } & 43 \mathrm{~m}^{2} / \mathrm{g} \\ \text { BET actual SAPG: } & 43 \mathrm{~m}^{2} / \mathrm{g} \\ \text { BET calc'd MSVD: } & 60 \mathrm{~nm} \\ \text { PPUV (from TEM PSD): } & 17,000 \\ \text { PPUV (all 60 nm particles): } & 9,000\end{array}$

The above numbers are representative of real values.

This is very good agreement, but the sample is an ideal polydispersed system. The two values of about $43 \mathrm{~m}^{2} / \mathrm{g}$ agree to within $0.08 \mathrm{~m}^{2} / \mathrm{g}$ of each other. Notice how using the BET MSVD value to calculate the PPUV generates a significant error. The TEM PSD data calculates to the same MSVD value of $60 \mathrm{~nm}$ and so it also has the same PPUV error. You are summing up a hypothetical monodispersed system. Using equation (5) and the measured polydispersed PSD data yields the correct answer, $\sim 17000$ primary particles/cubic micron.

In the real world of non-spherical particles, this set of BET measurements will not match because the primary particles will not be perfect spheres.

\section{Behavior of Primary Particle Size Distributions in Non-Ideal Mor- phologies}

The shapes of primary particles, the intergrowth between particles, deforming collisions of particles and the distance between primary particles will change all of these ideal conditions. Integrations of the surfaces and volumes of revolution ${ }^{4}$ of functions used to model nanoparticles will demonstrate these changes on MSVDs, BET and CTAB values. Since we have eliminated microporosity in our particles, BET values will equal CTAB values.

TEM number means are not involved in these changes because the measurements of diameters interactively will typically ignore intergrowth, etc. However, BET values will contain all the morphology changes including microporosity or "molecular roughness."

\section{The Effects of Intergrowth of Primary Particles on Specific Surface Area, BET and CTAB}

Figure 1 shows what is meant by the reinforcing intergrowth (RI) between primary particles. Point touching spheres represent $0 \%$ RI and the cylinder in the lower left represents $100 \%$ RI. RI then is the height in percent or decimal fraction of the final average radius of the material that fills in the gap between primary particles. This extra material is called intergrowth material or just intergrowth. A simple cylinder and sphere model is shown on the right side of Figure 1 along with a representation of real curved intergrowth. So, we can model this intergrowth or particle necking as two equal hemispheres and an intersecting bridging cylinder of radius RI in three dimensions.

Figure 3 shows that the absolute surface area (SA) drops to a low of $91.42 \%$ of the surface area of the starting spheres as RI increases from 0.0 to 0.7 . The surface area then increases to $100 \%$ at a RI of 1.0 (a cylinder). See Figures $1 \& 3$. Figure 3 shows graphically that the absolute surface area of a string of point contacting spheres is the same as the absolute surface area of the curved surface of a cylinder of equal diameter and height. This is not so obvious, but it is true and is shown at the ends of the plot. Intergrowth is the non-linear domain of the curve between the ends of the plot representing the two hemispheres and a cylinder.

However, this graph totally ignores specific surface area (SAPG) which is what we measure in our labs as BET nitrogen values. The SAPG of a sphere is higher than that of a cylinder because the cylinder simply weighs more than the sphere with equal absolute surface area. But, how does SAPG vary during these extremes of reinforcement on a weight or volume basis? 


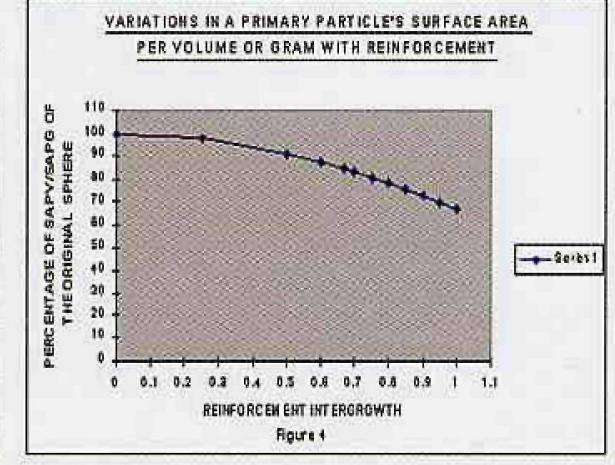

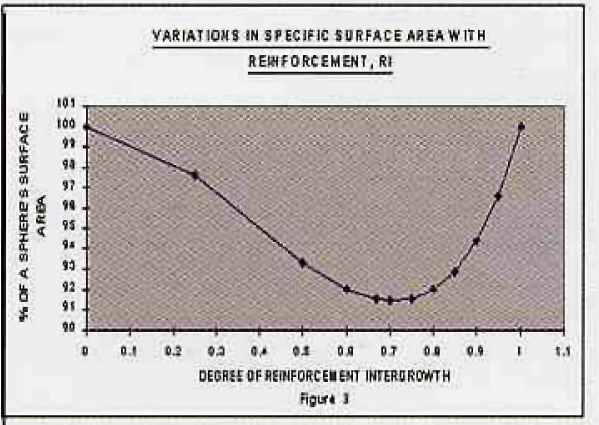

\section{Agglomerates and aggregates of primary particles}

This article is ignoring large aggregated and agglomerated structures. These larger porous clusters of primary particles are best handled by laser diffraction techniques. However, smaller welldispersed real aggregates are measured on-line in a TEM using an image analyzer set up to measure the projected area of aggregates. Calculating the equivalent circular diameter of a projected area has no bearing on primary particle MSVDs.

Concluding Remarks on Primary Particle Size Distributions Measured in a TEM

Figure 4 shows that surface area per gram (SAPG) starts at the high SAPG of a sphere (normalized to $100 \%$ here) and then gradually decreases to a low point representing $67 \%$ of the SAPG of the original spheres at 1.0 RI (a cylinder). There is no minimum or maximum between these two extremes of morphology. At a RI of 1.0 the original spheres have lost $33 \%$ of their SAPG and at that point, recognition of primary or ultimate particles is nearly impossible in a TEM. A string of spheres simply becomes a piece of spaghetti at $100 \%$ RI or $1.0 \mathrm{RI}$.

In Figure 5 are the plotted results of the integration of a sphere, a cylinder, and the cylindrical intergrowth of a spherical based system with increasing radii and RI. In all cases, the SAPG drops with increasing radius and RI. So any changes in 'morphology of particles' and particle sizes will change the SAPG values.

What is important here is that intergrowth, in particular, causes a change in SAPG, CTAB and BET specific surface area values. This then changes the value of the ideal MSVD calculated from, say, a BET value. Now let's look at the results of the integrations of spheres and cylinders where the length of the intergrowth cylinder exceeds the diameter of the hemispheres. What's important here is that the changes in the distance between primary particles also changes the SAPG values.

Deforming collisions between primary particles to form a non-circular cardioid shape lowers the SAPG values. Cardioids are graphed in polar coordinates and have the form:

$$
R=A-(B \times \cos (\theta)) \text {, where } A=B
$$

If $A<B$, you have a limacon shape.

How are you going to know what morphology you have, if you only have a 'faster-better-cheaper' BET value and a BET calculated MSVD? You can't. You need to do TEM PSD work to fully describe a primary particle size system and you need the BET value to calculate its MSVD for comparison.

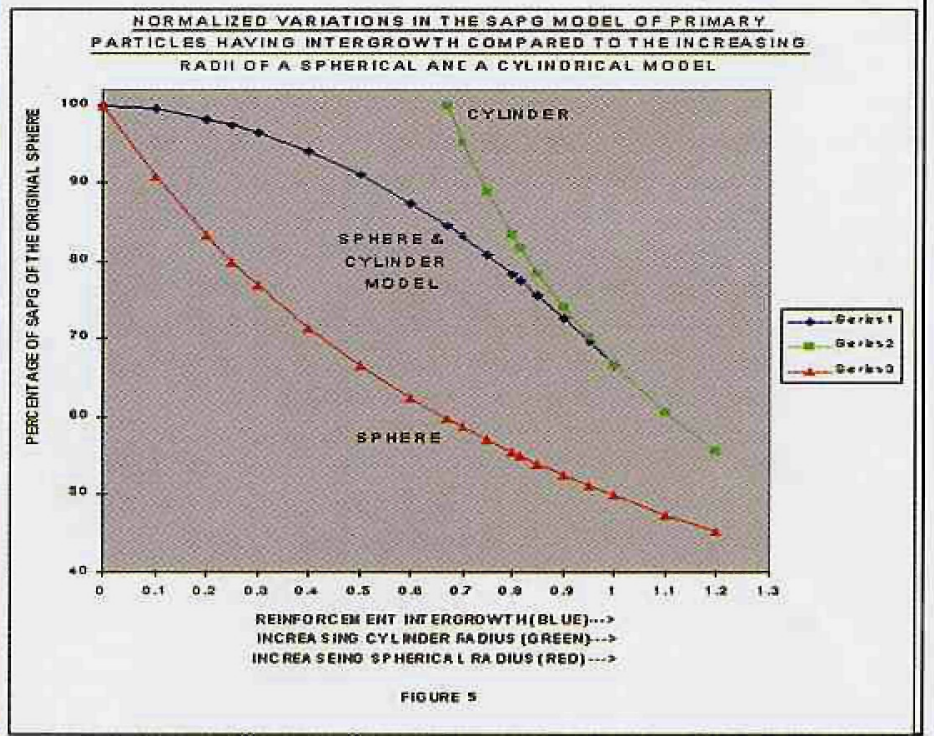

From the summing formulas, we can see that polydispersed non-ideal systems involve more detailed calculations than those based on simple geometric solids, monodispersed systems or the macro-determination of BET.

So here's the bottom line on intergrowth, collisions, irregular particle shapes and increasing distances between particles with necking. If the SAPG varies with morphology, then how can you tell what morphology you have from a BET value? You carit. You need to do the TEM image analysis work and to see a DIRECT OBSERVATION on your viewing screen or negative of what is going on in your sample. BET is a direct averaged observation of the INVISIBLE morphology of that same sample but also includes any non-ideal morphology, if present.

The BET value contains all the morphology differences. TEM diameters you measure do not account for these differences. So how can the BET and TEM calculated MSVDs be equal? They can't be in non-ideal systems. In an ideal monodispersed system, the TEM number mean, TEM MSVD, and BET MSVD are all equal. In non-ideal systems, the smallest value will be the TEM number mean. It will be the least affected by oversized reactor growth units. The next higher TEM value will be the calculated equivalent of an ideal BET MSVD. This TEM value is based on your interactive diameter measurements and does not include intergrowth. Thus, it is based on an ideal spherical system. This MSVD is greatly affected by outliers and reactor oversized material, if you counted them.

Now, the calculated value for MSVD based on BET can be lower, higher or equal to the TEM MSVD value. This is because the BET values depend on the morphologies of non-spherical primary particles you see in a TEM. Things like intergrowth and distances are BET MSVD wild card variables here.

So you should now have a better understanding of PSD, the mathematical tools to do a particle size distribution and/or to understand what your software program's PSD numbers are telling you. Calculus and analytical geometry modeling of the molecular roughness of the surfaces of primary particles is beyond the scope of this article, but is quite insightful. For example, I ran two samples of the same sized TiO, crystals by TEM. The crystals had no microporosity. However, one had triple the BET surface area. The $250 \mathrm{~nm}$ average primary particle size seen in the TEM did not change and the BET should have been $5.6 \mathrm{~m}^{2} / \mathrm{g}$, not $15 \mathrm{~m}^{2} / \mathrm{g}$. The real surface area value was 6.2 based on PSD. Be careful of those who read too much into BET numbers or calculating hypothetical sizes from BET values. Eliminating a TEM lab because of costs will eliminate your ability to directly image materials. TEM is your highest resolution referee in disputes over BET, aggregation, agglomeration, dispersion, porosity, structure and laser diffraction results of nanoscale particles. It takes an experienced microscopist and not an operator to sort all this out.

\section{Endnotes}

ASTM Method D3765-01 Standard Test Method for Carbon Blacks-CTAB (Cetyltrmethylammonium bromide) Surface Ared

${ }^{3}$ Allen, Terence, Particle Size Measuenent, $3^{\text {rd }}$ edition, Chapman and Hall, page 128 , 1981.

${ }^{4}$ Fisher Robert C. and Ziebur Allen D. Calcuilus and Analytical Geometry, Prentice-Hall Inc., $\mathrm{p} 251$ and $\mathrm{p} 442,1961$. 